\title{
Prevalência e qualidade de vida de escolares com hiperidrose primária no município de Aracaju-Sergipe
}

\author{
Prevalence and quality of life of schoolchildren with primary hyperhidrosis in the \\ municipality of Aracaju-Sergipe
}

\section{Prevalencia y calidad de vida de escolares con hiperhidrosis primaria en el municipio de Aracaju-Sergipe}

Maria Verônica dos Santos ${ }^{1}$, Maria Bernadete Galrão de Almeida Figueiredo ${ }^{1}$, Vanessa Rocha de Santana $^{1}$, Renata Lima Batalha de Andrade ${ }^{1}$, Cícera Sousa Silva ${ }^{1}$, Arthur Valido Deda ${ }^{2}$, Carla Viviane Freitas de Jesus ${ }^{1}$, Iris Sterfanie Santos ${ }^{2}$, Maria Júlia Nardelli ${ }^{1}$, Sônia Oliveira Lima ${ }^{1 *}$.

\section{RESUMO}

Objetivo: Estabelecer a prevalência de hiperidrose primária (HP) e sua interferência na qualidade de vida de estudantes, de uma escola de Aracaju-SE. Métodos: Estudo transversal, descritivo, exploratório e quantitativo com avaliação de 211 escolares de 8 a 17 anos do ensino fundamental e médio no período de abril a junho de 2019. Utilizou-se três questionários validados e autoaplicáveis que abordam os critérios de diagnóstico para HP, o grau de intensidade Hyperhidrosis Disease Severity Scale (HDSS), e a qualidade de vida em relação à HP. Os dados foram apresentados com média, desvio padrão, frequência absoluta e relativa. Utilizou-se o Qui-Quadrado de Pearson e Teste t de Student $(\mathrm{p}<0,05)$. Resultados: De 202 escolares inclusos, a prevalência da HP foi de $27,8 \%$, sem diferença entre o sexo e etnias. Ocorreu em sítios combinados e o sítio isolado palmar foi prevalente. O início dos sintomas deu-se na infância com níveis entre leve e moderada, com HDSS 2 e 3. Conclusão: A prevalência de HP foi alta, com caráter familiar significativo. Acomete, principalmente, as regiões palmo-plantar com início na infância, acarretando ansiedade, constrangimento, danos nas atividades escolares e nas relações interpessoais, aumentando em situações de tensão e preocupação, com impacto negativo na qualidade de vida.

Palavras-chave: Sudorese excessiva, Epidemiologia, Diagnóstico, Qualidade de vida.

\section{ABSTRACT}

Objective: Establish the prevalence of primary hyperhidrosis $(\mathrm{PH})$ and its interference in the quality of life of students, from a school of Aracaju-Se. Methods: Study cross-sectional descriptive, exploratory e quantitative whit evaluation of 211 schoolchildren of 8 the 17 years of elementary and high school in the period from April to June 2019. Three validated and self-administered questionnaires were used that address the diagnostic criteria for $\mathrm{PH}$, the degree of intensity of Hyperhidrosis Disease Severity Scale (HDSS), and a quality of life in relation to PH. The data were presented with average, standard deviation. absolute and relative freauencv. Used were Pearson's Chi-Sauare and Student's $t$-test $(p<0.05)$. Results: Of 202 schoolchildren included, the prevalence of $\mathrm{PH}$ was $27.8 \%$, with no difference between sex and ethnicity. It occurred in combined sites, and the isolated palmar site was prevalent. The onset of symptoms occurred in childhood with levels between mild and moderate, with HDSS 2 and 3. Conclusion: The prevalence of PH was high, with a significant family character. It mainly affects the palmoplantar regions beginning in childhood, causing anxiety, embarrassment, damage in school activities, and in interpersonal relationships, increasing in situations of tension and concern negative impact on quality of life.

Keywords: Excessive sweating, Epidemiology, Diagnosis, Quality of life.

\section{RESUMEN}

Objetivo: Estabelecer la prevalência de hiperhidrosis primaria (HP) y su interference em la calidad de vida de los estudiantes, de uma escuela de Aracaju-Se. Métodos: Estudio transversal, descriptivo, exploratório y cuantitativo com evaluación de 211 escolares de 8 a 12 años de primaria y secundaria em el período de abril a junio de 2019. Se utilizaron três cuestionarios validados y autoadministrados que abordan los critérios diagnósticos de HP, el grado de intensidad de la escala de gravedad de la enfermedad de hiperhidrosis (HDSS) y la calidad de vida em relación com la HP. Los datos se presentaon com media, desviación estândar, frecuencia absoluta y relativa. Se utilizaron las pruebas de chi-cuadrado e Pearson y T de Student. Resultados: De 202 escolares incluidos, la prevalencia de HP fue de 27,8\%, sin diferencia entre sexo y etnia, se presentó en sitios combinados y predominó el sitio palmar aislado. El inicio de los síntomas se produjoen la infancia com niveles entre leves y moderados, com HDSS 2 y 3 . Conclusión: La prevalência de HP fue alta, com um caráter familiar significativo. Afecta, principalmente, a las regiones palmoplantar, comenzando em la infância, provocando ansiedade, verguenza, daño em las atividades escolares y em las relaciones interpersonales, aumentando em situaciones de tensión y preocupación, com impacto negativo em la calidad de vida.

Palabras clave: Sudoración excesiva, Epidemiología, Diagnóstico, Calidad de vida.

1 Universidade Tiradentes (UNIT), Aracaju - SE. *E-mail: sonialima.cirurgia@gmail.com

2 Universidade Federal de Sergipe, São Cristóvão - SE. 


\section{INTRODUÇÃO}

O suor é fundamental para manutenção da temperatura corporal, principalmente durante os exercícios e, quando excessivo, é considerado como hiperidrose (FERREIRA FG, et al., 2017). A literatura classifica a hiperidrose como primária e secundária, com apresentação nas formas difusas e focais. Na Hiperidrose Primária (HP) a sudorese é localizada, simétrica, afeta principalmente axilas, palma da mão, face e planta dos pés, com início dos sintomas na infância, adolescência e fase adulta, podendo haver ligações com fatores genéticos (SILVA SLS, et al., 2017; MURARO CSP, et al., 2019). A intensidade da HP varia de leve a grave, e a sudorese incontrolável pode acarretar complicações secundárias como o surgimento de bromidrose $87 \%$, infecção fúngica $31 \%$ e entorses $43 \%$ (LIMA SO, et al., 2017).

A idade específica do início dos sintomas depende da localização anatômica, sendo principalmente a HP palmar com início na infância, a axilar na adolescência e a crânio facial na idade adulta (FENILI R, et al., 2009). A Hiperidrose Secundária (HS) é decorrente de doenças como hipertireoidismo, menopausa e distúrbios hormonais que desencadeiam aumento da sudorese (FENILI R, et al., 2009; MURARO CSP, et al., 2019).

A etiologia da HP é desconhecida e associa-se a intenso estresse emocional, ocupacional e social (SAKIYMA PY, et al., 2012). Os dados epidemiológicos evidenciam que a incidência na população está compreendida entre 0,6\% a 16,7\%, acometendo homens e mulheres na mesma proporção (LIMA SO, et al., 2015; LIMA SO, et al., 2018; LIMA SO, et al., 2019).

Na primeira infância, o suor excessivo interfere no equilíbrio da criança e os pais sentem dificuldades em lidar com os questionamentos sobre a doença, causando transtornos aos familiares. Na fase escolar, o portador de HP sofre bullying, refletindo em isolamento social e tristeza (LIMA SO, et al., 2020). O suor excessivo e incessante é uma situação desesperadora, causa transtornos nas atividades diárias e compromete o desempenho no trabalho e nas relações sociais, propiciando às pessoas que têm esse agravo, constrangimento, angústia e ansiedade (BRAGANÇA GMG, et al., 2014; BARROS AC, et al., 2020).

O diagnóstico da HP é clínico, com base nos seguintes critérios: apresentar suor excessivo, localizado e visível por pelo menos seis meses de duração e sem causa aparente. Associado à presença de pelo menos dois desses aspectos: suor bilateral e relativamente simétrico, ocorrendo no mínimo uma vez por semana, danos nas atividades diárias, com início antes dos 25 anos, antecedentes familiares, cessação de suor durante o sono, piora com o estresse e sem ou com pouca interferência da temperatura (FENILI R, et al., 2009).

Com o intuito de promover o melhor tratamento, com menos efeitos colaterais aos pacientes que possuem a HP, estudos vêm sendo desenvolvidos e o tratamento pode ser clínico ou cirúrgico. $O$ tratamento clínico da HP pode ser oral, tópico ou mediante injeção de toxina botulínica (WOLOSKER N e FAKUDA JM, 2015). O tratamento cirúrgico pode ser realizado pela ressecção das glândulas sudoríparas écrinas ou por simpatectomias torácica e lombar (LIMA SO, et al. 2012; WOLOSKER N e FAKUDA JM, 2015; LIMA SO, et al. 2017).

A hiperidrose primária não é considerada uma doença grave quanto à morbidade e mortalidade, porém provoca elevados índices de ansiedade, estresse e apreensão, além de comprometer determinadas profissões. Devido ao impacto negativo que a HP pode causar na qualidade de vida do paciente afetando as áreas emocional, social e profissional, é importante o conhecimento e divulgação dessa doença para a população em geral (FIORELLI RKA, et al., 2011; BRAGANÇA GMG, et al., 20014; DIAS LIN, et al., 2016; LIMA SO, et al., 2019; LIMA SO, et al., 2020).

Nessa perspectiva, objetivou-se estabelecer a prevalência e o perfil de estudantes de uma escola no município de Aracaju/SE, portadores de hiperidrose primária e sua interferência na qualidade de vida.

\section{MÉTODOS}

Trata-se de um estudo transversal, de caráter descritivo e exploratório com abordagem quantitativa, direcionado a estudantes do ensino fundamental 1,2 e de ensino médio de uma escola localizada no município de Aracaju/SE, desenvolvido entre os meses de abril e junho de 2019. Foram incluídos os 
questionários de estudantes de ambos os sexos com faixa etária dos 8 aos 17 anos, e excluídos os questionários com preenchimento inadequado e/ou com o Termo de Consentimento Livre e Esclarecido não assinado.

Utilizou-se a fórmula de Pocock para o cálculo da amostra mínima de alunos, estimando prevalência de $50 \%$, margem de erro de $5 \%$ e intervalo de confiança de $95 \%$. A escola, selecionada para o estudo, possuía na faixa etária pesquisada 500 alunos matriculados, portanto, a amostra mínima calculada foi de 205 estudantes.

Para coleta de dados foram utilizados três instrumentos: o primeiro correspondeu ao Questionário de Critérios Diagnósticos (FELINI R, et al., 2009), utilizado para verificar se há ou não presença de HP, de acordo com as manifestações clínicas da doença. Deve ser assinalado "sim" ou "não" para a presença de sudorese excessiva e, os que marcaram "sim", devem assinalar pelo menos, mais duas características relacionadas à HP. Aqueles que responderam "não" ou os que colocaram "sim", mas não marcaram duas ou mais características relativas à doença, não responderam aos questionários seguintes.

O segundo correspondeu ao Hyperhidrosis Disease Severity Scale (HDSS) que possui o objetivo de avaliar o grau da HP. Os graus 01 e 02 são considerados leve/moderado e os graus 03 e 04 são considerados graves (VARELLA AYM, et al., 2016). E o terceiro representou o Questionário de Qualidade de Vida (QdV) devido à HP (CAMPOS JRM, et al., 2003), que trata da percepção dos portadores de HP sobre qualidade de vida em quatro domínios principais: funcional-social, pessoal, emocional e condições especiais, classificados em cinco níveis de satisfação dos pacientes, obtidos através de escores. A escala varia com intervalo de vinte a cem pontos: quando o total for maior que 84 , a QV é considerada muito ruim; 68-83, ruim; 52-67, boa; 36-51, muito boa, e 20-35, excelente.

Realizou-se divulgação e palestra por professional médico expert em HP a respeito do projeto da pesquisa e da doença Hiperidrose Primária, direcionada aos coordenadores e professores da escola avaliada. Houve prévia capacitação dos educadores para a entrega aos alunos e para busca ativa dos formulários com os questionários que foram utilizados na pesquisa, assim como o Termo de Consentimento Livre e Esclarecido (TCLE).

Os questionários enviados para as residências foram preenchidos e assinados pelos responsáveis dos escolares, por serem menores de 18 anos. Todos os participantes que responderam "sim" para presença de suor excessivo, localizado e visível, por pelo menos seis meses de duração e sem causa aparente, completaram o questionário de diagnóstico para HP e responderam, também, aos questionários da escala HDSS e sobre qualidade de vida.

Os dados foram tabulados e analisados para calcular a prevalência da HP entre os estudantes e foi utilizado o percentual do somatório dos participantes que assinalaram "sim" e em, pelo menos dois itens, no Questionário de Critérios Diagnósticos dividido pelo total de entrevistados para cada grupo de sim ou não. Nos demais dados, aplicou-se a estatística básica: média, desvio padrão, frequência absoluta e relativa, bem como análises paramétricas de Qui-Quadrado de Pearson e Teste t de Student a um nível de significância de $p<0,05$.

A pesquisa teve aprovação do Comitê de Ética em Pesquisa da Universidade Tiradentes, com processo nํ 3.266630. Foram seguidas as diretrizes da Resolução n 510/16 do Conselho Nacional de Saúde. Para todos os participantes foi apresentado o TCLE que assegurava o sigilo e proteção da imagem, inclusive com a não identificação, além das informações sobre o tema, objetivos e métodos utilizados na pesquisa.

\section{RESULTADOS}

Foram coletados 211 questionários, sendo 9 (4,3\%) excluídos por preenchimento inadequado. Dentre os 202 entregues devidamente preenchidos, 106 (52,4\%) discentes eram do sexo feminino e 96 (47,6\%) do masculino. Destes, 56 (27,8\%) afirmaram possuir critérios para o diagnóstico de HP, sendo 2 (3,57\%) com diagnóstico prévio confirmado por médico. Dos portadores de HP, 28 (50\%) eram do sexo feminino com prevalência de 26,4\% e 28 (50\%) do masculino com prevalência de 29,2\% em relação aos 202 escolares avaliados (Tabela 1). 
Tabela 1 - Frequências absoluta e relativa dos critérios diagnósticos da HP nos discentes de uma escola no município de Aracaju/SE, no ano de $2019(\mathrm{n}=56)$.

\begin{tabular}{lcc}
\hline \multicolumn{1}{c}{ Critérios Diagnósticos de HP } & N & Frequência \\
\cline { 2 - 3 } Suor bilateral e relativamente simétrico & 31 & 55 \\
Frequência de pelo menos uma vez por semana & 24 & 43 \\
Prejuízo nas atividades diárias & 30 & 54 \\
Idade de início menor que 25 anos & 49 & 88 \\
História familiar positiva & 20 & 36 \\
Cessação do suor durante o sono & 19 & 34 \\
Piora em situações de estresse & 18 & 32 \\
Sem ou pouca interferência de temperatura & 21 & 38 \\
\hline
\end{tabular}

Fonte: Santos MV, et al., 2020.

A faixa etária dos escolares foi entre 8 e 17 anos, apresentando média idade de 11,67 anos e desvio padrão (DP) 2,38 (Figura 1). Em todas as faixas etárias avaliadas foram identificados indivíduos com HP (Figura 2), cuja média foi de 12 anos e o desvio padrão de 2,75.

Figura 1 - llustração da relação entre a frequência relativa em relação idade dos escolares avaliados no município de Aracaju/SE, no ano de 2019 ( $\mathrm{n=202).}$

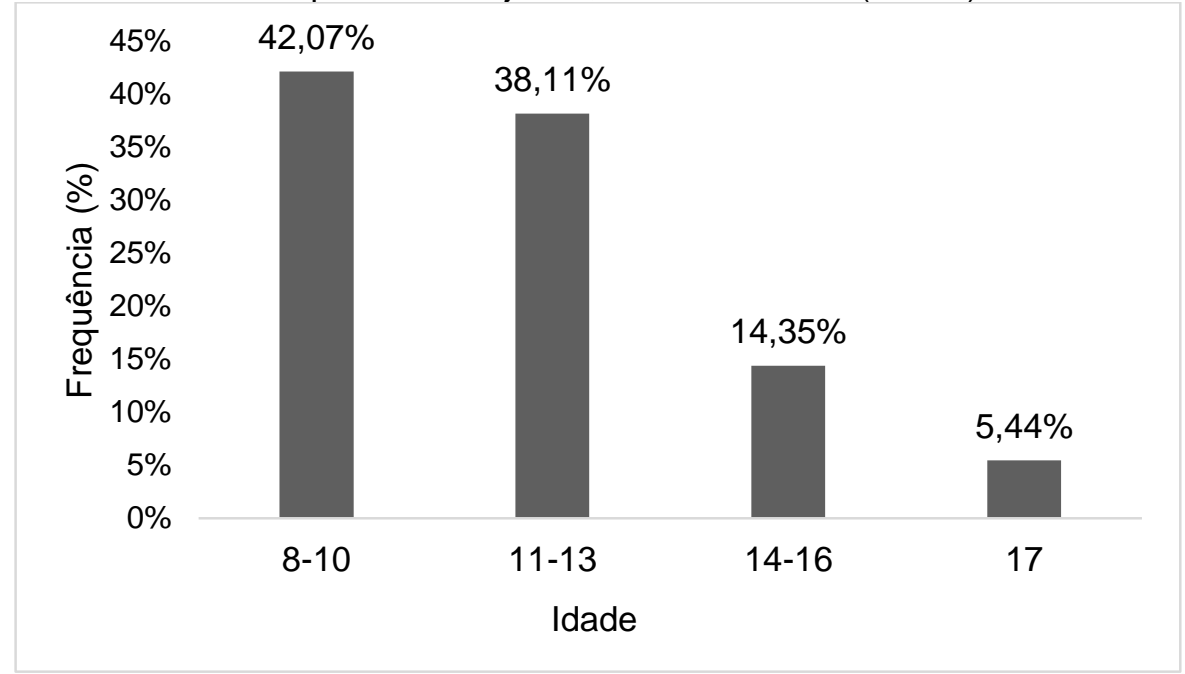

Fonte: Santos MV, et al., 2020.

Figura 2 - Prevalência Hiperidrose Primária em relação a idade dos escolares no município de Aracaju/SE, no ano $2019(n=56)$.

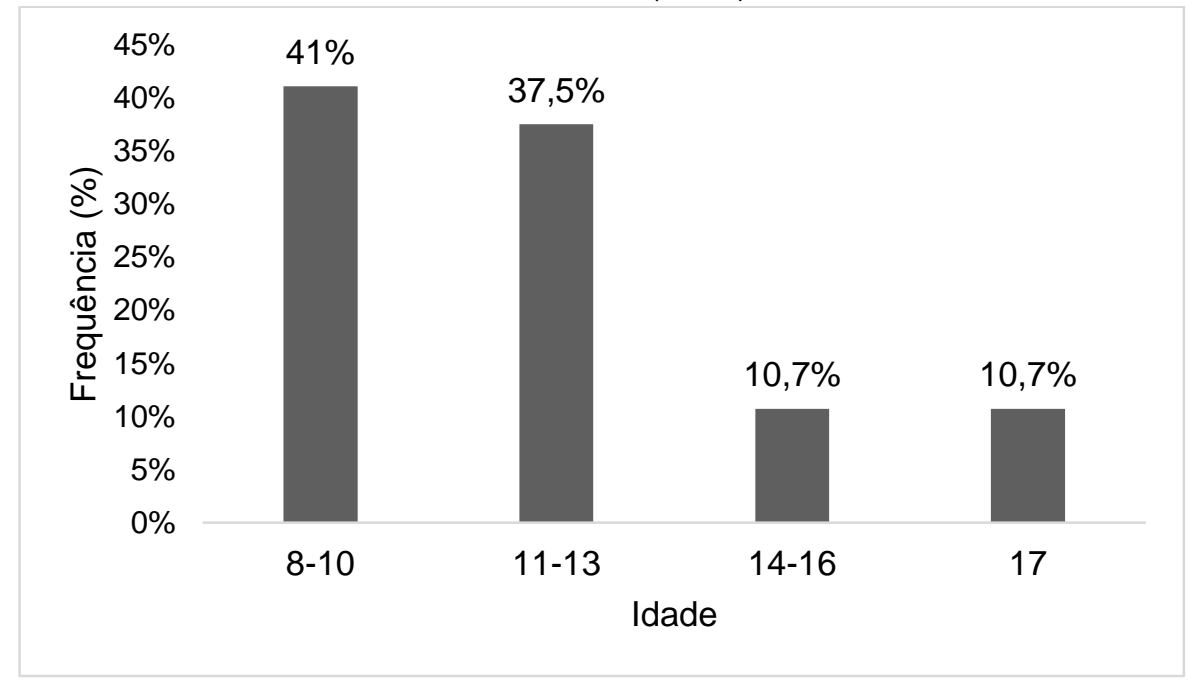

Fonte: Santos MV, et al., 2020. 
Não houve diferença em relação a etnia (Tabela 2) e a região corporal que predominou; o suor excessivo foi o palmar, seguido do facial, plantar e axilar $(p<0,001)$. Na associação das regiões o destaque foi a palmarplantar com 9\%, seguido das regiões crânio- palmar e axilar-palmar ambas com 7\%, e da associação crânio, face, palmar e plantar com 5\%. De forma geral, as associações crânio-plantar e face-axilar apresentaram os menores índices $2 \%$ nos escolares que participaram do estudo (Tabela 3).

Tabela 2 - A frequência absoluta e relativa relacionada a etnia dos escolares com HP, no município de Aracaju/SE, no ano de 2019.

\begin{tabular}{|c|c|c|c|c|c|}
\hline \multirow{2}{*}{ Etnia } & \multicolumn{2}{|c|}{ População Total } & \multicolumn{2}{|c|}{ Hiperidrose } & \multirow{2}{*}{ Valor $p$} \\
\hline & $\mathbf{N}$ & $\%$ & $\mathbf{N}$ & $\%$ & \\
\hline Amarelo & 4 & 2,0 & 2 & 3,5 & \multirow{4}{*}{0,4489} \\
\hline Branco & 63 & 31,2 & 15 & 26,7 & \\
\hline Negro & 11 & 5,5 & 6 & 10,7 & \\
\hline Pardo & 124 & 61,4 & 33 & 58,9 & \\
\hline
\end{tabular}

Fonte: Santos MV, et al., 2020.

Tabela 3 - Frequências absoluta e relativa das regiões acometidas pela HP nos escolares do município de Aracaju/SE, no ano de $2019(n=56)$.

\begin{tabular}{|c|c|c|c|}
\hline \multirow{2}{*}{ Regiões Acometidas } & \multicolumn{2}{|c|}{ Frequência } & \multirow{2}{*}{ Valor $p$} \\
\hline & $\mathbf{N}$ & $\%$ & \\
\hline Palmar & 17 & 30 & \\
\hline Facial & 8 & 14 & \\
\hline Plantar & 6 & 11 & \\
\hline Axial & 5 & 9 & \\
\hline Palmar + Plantar & 5 & 9 & \\
\hline Crânio + Palmar & 4 & 7 & $<0,001$ \\
\hline Axilar + Palmar & 4 & 7 & \\
\hline Crânio + Face + Palmas + Plantar & 3 & 5 & \\
\hline Crânio & 2 & 4 & \\
\hline Crânio + Plantar & 1 & 2 & \\
\hline Face + Axilar & 1 & 2 & \\
\hline
\end{tabular}

Fonte: Santos MV, et al., 2020.

Quanto ao surgimento dos sintomas de HP, 27 (48,2\%) discentes referiram ter iniciado entre 5-10 anos, 10 (17,85\%) entre 10-15 anos, 8 (14,3\%) entre 0-5 anos, 4 (7,1\%) de 15-17anos e 7 (12,5\%) não souberam informar a idade de início dos sintomas.

Ao avaliar prejuízos nas atividades diárias, 29 (51,8\%) escolares destacaram a sudorese excessiva interferindo nas áreas psíquica, social e estudantil, com influência em 37,9\% nas brincadeiras, 3,4\% no manuseamento de livros, $20,6 \%$ na apresentação de trabalhos $24 \%$ e na escrita. Dessa forma, provocando ansiedade em $13,7 \%$ dos escolares, vergonha em 6,9\% e constrangimento em 6,9\%. No que concerne à intensidade, $56,5 \%$ dos escolares referiam leve, 37,5\% moderado e 8,9\% intenso. De acordo com a escala de gravidade HDSS, a maioria se enquadrou nos Grau 2, seguidos dos graus 3, 1 e 4 ( $p=0,0013)$ (Tabela 4).

Tabela 4 - Frequência de respostas em relação a escala de gravidade da hiperidrose (HDSS), de escolares com HP, no município de Aracaju/SE, no ano de $2019(n=56)$.

\section{Escala de Gravidade da Hiperidrose}

\begin{tabular}{llccc}
\hline Grau 1 & Imperceptível e nunca interfere nas atividades diárias & 8 & 14,2 & \\
Grau 2 & Tolerável e interfere nas atividades diárias & 22 & 39,3 & \multirow{2}{*}{0,0013} \\
Grau 3 & Pouco tolerável e interfere constantemente nas atividades diárias & 16 & 28,5 & \\
Grau 4 & Intolerável e interfere sempre nas atividades diárias & 4 & 7,1 & \\
& Não Informado & 6 & 10,7 & \\
\hline
\end{tabular}

Fonte: Santos MV, et al., 2020. 
Nos discentes que autoafirmaram serem portadores de HP, a qualidade de vida foi avaliada com o questionário baseado em domínios funcional-social, pessoal, emocional, próprio e condições especiais, associados aos escores de 1 a 5, sendo 1 considerada excelente, 2 muito boa, 3 boa, 4 ruim e 5 muito ruim. Dentre os domínios, os das condições especiais apresentou maior pontuação, com 75 pontos no quesito ambiente fechado/quente equivalendo a $27 \%$ dos alunos e, 60 pontos, no quesito tenso ou preocupado, constituindo $27 \%$ dos discentes. Na sequência, o domínio emocional ou outros, com 55 pontos no item rejeição, equivalente a $20 \%$ dos entrevistados. O domínio funcional social, 54 pontos, no trabalho manual com frequência relativa de $32 \%$. Por último, no domínio pessoal, 36 pontos no item brincar com colegas, com abrangência de $21 \%$ dos discentes (Tabela 5). A média dos domínios variou de 4,78 a 17,18 com desvio padrão de 3,09 a 6,26.

Tabela 5 - Percepção dos alunos que responderam aos critérios diagnósticos quanto a Qualidade de Vida.

\begin{tabular}{|c|c|c|c|c|c|c|c|c|c|c|}
\hline $\begin{array}{l}\text { DOMÍNIOS } \\
\text { FUNCIONAL SOCIAL }\end{array}$ & \multicolumn{2}{|c|}{$\begin{array}{c}\text { EXCELENTE } \\
1 \text { ponto } \\
\mathrm{n}(\%)\end{array}$} & \multicolumn{2}{|c|}{$\begin{array}{l}\text { MUITO } \\
\text { BOA } \\
2 \text { pontos } \\
\text { n (\%) }\end{array}$} & \multicolumn{2}{|c|}{$\begin{array}{c}\text { BOA } \\
3 \text { pontos } \\
\text { n (\%) }\end{array}$} & \multicolumn{2}{|c|}{$\begin{array}{c}\text { RUIM } \\
4 \text { pontos } \\
\text { n (\%) }\end{array}$} & \multicolumn{2}{|c|}{$\begin{array}{l}\text { MUITO } \\
\text { RUIM } \\
5 \text { pontos } \\
\text { n (\%) }\end{array}$} \\
\hline Escrever & 20 & (36) & 18 & (32) & 13 & (23) & 4 & (7) & 2 & (4) \\
\hline Trabalhos manuais & 22 & (39) & 9 & $(16)$ & 18 & (32) & 5 & (9) & 4 & (7) \\
\hline Passatempo predileto & 20 & $(36)$ & 20 & (36) & 9 & $(16)$ & 5 & (9) & 2 & (4) \\
\hline Prática de esportes & 24 & $(43)$ & 9 & $(16)$ & 13 & (23) & 5 & (9) & 3 & (5) \\
\hline Segurar objetos & 27 & $(48)$ & 16 & (29) & 6 & $(11)$ & 4 & (7) & 2 & (4) \\
\hline Apertar mãos (pessoas) & 30 & $(54)$ & 7 & (13) & 8 & $(14)$ & 5 & (9) & 4 & (7) \\
\hline Estar com amigos (lugares públicos) & 15 & $(27)$ & 12 & $(21)$ & 7 & (13) & 0 & $(0)$ & 5 & (9) \\
\hline Dançar socialmente & 19 & $(34)$ & 12 & $(21)$ & 10 & $(18)$ & 9 & $(16)$ & 5 & (9) \\
\hline \multicolumn{11}{|l|}{ PESSOAL $^{2}$} \\
\hline Segurar as mãos & 27 & $(48)$ & 11 & (20) & 12 & $(21)$ & 5 & (9) & 2 & (4) \\
\hline Tocar os familiares & 32 & $(57)$ & 14 & $(25)$ & 6 & $(11)$ & 4 & (7) & 2 & (4) \\
\hline Brincar com os colegas & 17 & $(30)$ & 14 & $(25)$ & 12 & $(21)$ & 4 & (7) & 7 & $(13)$ \\
\hline \multicolumn{11}{|l|}{ EMOCIONAL OU COM OUTROS ${ }^{3}$} \\
\hline Ter que se justificar & 20 & (36) & 9 & (16) & 9 & (16) & 7 & $(13)$ & 8 & $(14)$ \\
\hline Outros demonstram rejeição & 16 & $(29)$ & 5 & (9) & 12 & $(21)$ & 6 & $(11)$ & 11 & $(20)$ \\
\hline \multicolumn{11}{|l|}{ CONDIÇÕES ESPECIAIS ${ }^{4}$} \\
\hline Em ambientes fechados ou quentes & 10 & $(18)$ & 5 & (9) & 14 & (25) & 11 & $(20)$ & 15 & (27) \\
\hline Tenso ou preocupado & 8 & $(14)$ & 10 & $(18)$ & 8 & $(14)$ & 15 & $(27)$ & 11 & $(20)$ \\
\hline Pensando no assunto & 17 & $(30)$ & 13 & $(23)$ & 12 & $(21)$ & 5 & (9) & 4 & (7) \\
\hline Em avaliação ou falando em público & 15 & $(27)$ & 10 & $(18)$ & 14 & $(25)$ & 11 & $(20)$ & 5 & (9) \\
\hline Usando sandálias ou descalço & 16 & $(29)$ & 10 & $(18)$ & 12 & $(21)$ & 8 & $(14)$ & 8 & $(14)$ \\
\hline Usando roupas coloridas & 27 & $(48)$ & 6 & $(11)$ & 12 & $(21)$ & 5 & (9) & 5 & (9) \\
\hline Problemas na escola ou serviço & 22 & $(39)$ & 11 & $(20)$ & 10 & $(18)$ & 8 & $(14)$ & 4 & $(7)$ \\
\hline
\end{tabular}

Legenda: ${ }^{1}$ Média 15,34, Desvio Padrão 6,18; ${ }^{2}$ Média 5,91, Desvio Padrão 3,68; ${ }^{3}$ Média 4,78, Desvio Padrão 3,09; ${ }^{4}$ Média 17,8, Desvio Padrão 6,26.

Fonte: Santos MV, et al., 2020.

O Teste Qui-Quadrado foi utilizado para avaliar se a qualidade de vida e os fatores associados à HP tinham relações de dependência ou independência. $O$ valor de $p<0,05$, evidencia a rejeição da independência entre as variáveis qualidade de vida e fatores associados à HP ao nível de $5 \%$ de significância, isto é, a qualidade de vida e os fatores possuem relação de dependência. 
O Teste t de Student verificou, em todos os casos, que os valores $p$ são maiores que a estatística teste (valor T) então, a um nível de significância de $5 \%$, não rejeitamos a hipótese nula de que a média das variáveis estão próximas dos valores nominais $(12 ; 15 ; 6 ; 4 ; 17$, respectivamente) (Tabela 6).

Tabela 6 - Teste t de Student para uma amostra.

\begin{tabular}{lccc}
\hline & Valor T & Graus de Liberdade & P-valor \\
\hline Idade & 0 & 55 & 1 \\
Domínio Funcional-Social & $-0,0009$ & 55 & 0,9993 \\
Domínio Pessoal & 0,0217 & 55 & 0,9827 \\
Domínio Emocional & $-0,0088$ & 55 & 0,993 \\
Domínio Condições Especiais & 0,2135 & 55 & 0,8317 \\
\hline
\end{tabular}

Fonte: Santos MV, et al., 2020.

\section{DISCUSSÃO}

A HP é uma doença benigna que tem influência negativa na qualidade de vida dos pacientes, no entanto, são poucos os estudos que mostram o número de indivíduos acometidos por essa doença estigmatizante. De acordo com os dados epidemiológicos, observa-se grande variação de $0,93 \%$ a $38 \%$ na prevalência da HP, na população mundial (MUTHSAMY A, et al., 2016; HASIMOTO EM, et al. 2018).

No presente estudo, a prevalência de HP foi de $27,8 \%$ superior a algumas pesquisas realizadas no Brasil com acadêmicos de Medicina portadores de HP. Lima SO, et al. (2015) e Lima SO, et al. (2019) em Aracaju/SE apresentaram prevalência de $14,57 \%$ e $18 \%$, respectivamente. Westphal FL, et al. (2011) de 5,5\% em Manaus/AM. Morard DKT, et al. (2019) encontrou incidência de 20,56\%, também em acadêmicos de Medicina de universidades do Rio de Janeiro/RJ. Enquanto, que, em pesquisa realizada com estudantes universitários na Índia, Muthsamy A, et al. (2016) encontraram 38\% de HP. Esses autores consideraram que essa frequência elevada, comparada à mundial, pode estar relacionada à subjetividade da avaliação e à utilização de uma determinada faixa etária com uma amostra específica.

Hasimoto EN, et al. (2018) avaliaram 4.133 residentes da cidade de Botucatu e identificaram 2,07\% de suor excessivo entre os entrevistados e, após a avaliação médica, a prevalência de HP passou para 0,93\%. Fenilli R, et al. (2009) inferem sobre a possibilidade de metodologias diferentes influenciarem na divergência de dados estatísticos. Lima SO, et al. (2015) ressaltam que se as entrevistas fossem presenciais, individualmente e conduzidas por pessoas especializadas, a prevalência da HP seria mais fidedigna.

Não houve diferença de HP entre os sexos, concordando com outros estudos (FENILLI R, et al., 2009; LIMA SO, et al., 2018; LIMA SO, et al., 2019). Em relação a idade dos escolares de 8 a 17 anos portadores de HP, a média foi de 12 anos, com maior frequência de início, entre 5 e 10 anos (48,2\%), corroborando com os estudos de Toscano L, et al. (2019) em que a idade média de início dos sintomas foi aos 10 anos. Estudos reforçam o surgimento dos sintomas antes dos 25 anos (ESTEVAN FA, et al., 2017; LIMA SO, et al., 2020). Portanto, a HP é uma doença crônica que inicia na fase de desenvolvimento psíquico social do indivíduo, podendo interferir negativamente nas suas atividades diárias.

Não houve diferença estatística em relação a etnia, porém a presença de hiperidrose primária foi mais referida entre os pardos, semelhante a outros estudos (LIMA SO, et al., 2015; BARROS AC, et al., 2020), achados que podem ser influenciados pela miscigenação das raças. No que concerne a história familiar, foi indicada como positiva por 35,7\% dos escolares, em consonância com (Tu Y-R, et al. 2007; HASIMOTO EN, et al., 2018; MIRKOVIC SE, et al., 2018; LIMA SO, et al., 2020). Segundo Ro KM, et al. (2002), a HP é uma doença com forte influência genética, cujo os sítios palmar e plantar têm caráter autossômico dominante.

No que se refere aos sítios isolados, o palmar apresentou maior prevalência e o padrão associado de maior acometimento foi palmar-plantar, semelhante aos estudos de Lima SO, et al., 2015; Lima SO, et al., 2019; Hasimoto EN, et al., 2018; Miotto A, et al., 2018; Lima SO, et al., 2019; Estevan FA, et al., 2017. O presente estudo mostrou maior prevalência de sítio isolado em relação aos associados, enquanto a literatura pertinente mostra predomínio no acometimento das regiões associadas 74,4\% e 64,9\% em relação a isolada $25,6 \%$ e $35,1 \%$, respectivamente (REIS GDD, et al., 2011; SILVA SLS, et al., 2017). 
Dias LIN, et al. (2016) apresentaram prevalência de sudorese excessiva na associação das regiões axilarpalmar (54\%). Moraes DKT e Rocha GK (2018) verificaram que $29 \%$ dos escolares apresentaram sudorese excessiva nos pés e Horn G (2015) encontrou acometimento de $70 \%$ na região axilares. De acordo com Moraes DKT e Rocha GK (2018) a região mais afetada depende da faixa etária, desde que na HP palmar e plantar os sintomas iniciam na infância, na HP axilar na puberdade e a HP craniofacial na vida adulta. Portanto, as diferenças nas frequências da HP em diferentes sítios podem ser explicadas pela amostra pesquisada.

Em relação ao impacto nas atividades diárias, os piores índices estavam relacionados ao cotidiano estudantil como escrever, manusear livros e apresentar trabalhos, fato explicado por ter sido a região palmar a mais acometida pela HP. Lima SO, et al. (2015); Lima SO, et al. (2019) verificaram que a HP causa constrangimentos em estudantes de Medicina por interferir em suas atividades acadêmicas. Barros AC, et al. (2020) relataram que a sudorese excessiva é causa de constrangimento, ansiedade e vergonha, em prestadores de serviço da área de saúde. Assim, percebe-se que é importante o diagnóstico e tratamento precoce da HP com o intuito de evitar o constrangimento dos seus portadores, desde o ensino fundamental até a vida professional.

Constatou-se a prevalência da HP leve 30 (53,5\%) e HP moderada 21 (37,5\%) com um percentual relevante interferindo, frequentemente ou sempre, nas atividades diárias. Na escala HDSS verificou-se também, que $44(80 \%)$ dos estudantes referiram que o suor interferia nas suas atividades diárias, e os graus pouco tolerável e intolerável ocorreram em 20 (35,7\%) desses. A literatura pertinente apresenta diversidade na intensidade da sudorese porém, em todos os estudos, os graus que interferem nas atividades diárias e são intoleráveis estão presentes (DOOLITTLE J, et al., 2016; SILVA SLS, et al., 2017; LIMA SO, et al., 2019; TOSCANO P, et al., 2019). Dessa forma, há necessidade de intensificar a divulgação sobre a existência da doença HP inferindo o seu tratamento que pode ser realizado desde a infância, melhorando a qualidade de vida dos seus portadores.

$\mathrm{Na}$ avaliação da QdV verificou-se que, entre os escolares, a máxima pontuação foi no domínio condições especiais, nos quesitos ambiente fechado e quente e no tenso e preocupado. No domínio funcional social, os escolares relataram apresentar maior incomodo para realizar trabalhos manuais. No domínio pessoal, o item brincar com os colegas apresentou a pior pontuação e, no domínio emocional, o item rejeição. Hasimoto EN, et al. (2018); Lima SO, et al. (2019); Westphal FL, et al. (2011) também verificaram maior pontuação nos itens ambiente fechado e quente e no tenso e preocupado.

Vasconcelos CFM, et al. (2020) verificaram pior QdV no domínio funcional. Lima SO, et al. (2019) e Westphal FL, et al. (2011) verificaram que os piores índices no domínio condição especial foi dançar socialmente, no domínio pessoal foi segurar a mão e no domínio emocional foi justificar o suor. As diferenças encontradas nos domínios se devem a distintas faixas etárias das populações estudadas, em cada pesquisa, porém é perceptível a inferência negativa da HP na qualidade de vida de seus portadores em qualquer faixa etária acometida.

A limitação do presente estudo foi a não confirmação do diagnóstico de hiperidrose primária por especialistas. No entanto, teve a importância de propagar o conhecimento da hiperidrose primária no ambiente escolar, com o intuito de mostrar aos professores e responsáveis dos menores portadores de HP os estigmas dessa doença crônica. Dessa forma auxiliando na interação social, melhorando a autoestima e a qualidade de vida, dos portadores de HP, reduzindo o preconceito da sociedade sobre essa doença estigmatizante.

\section{CONCLUSÃO}

A prevalência de HP em escolares do ensino fundamental e médio, no município de Aracaju/SE, é de $27,8 \%$, sem diferença entre os sexos, etnias, com caráter familiar significativo. Acomete, principalmente, as regiões palmar e palmar-plantar e os primeiros sintomas ocorrem na infância e adolescência, acarretando ansiedade, constrangimento, danos nas atividades escolares e nas relações interpessoais. A HP com intensidade leve e moderada e HDDS 2 e 3 foram prevalentes com impacto negativo na QdV principalmente quando refere estar em ambiente fechado/quente e em situações de tensão e preocupação. 


\section{REFERÊNCIAS}

1. BARROS AC, et al. Impacto da hiperidrose nas atividades laborais da enfermagem em um hospital de urgência de Sergipe. Revista Online de Pesquisa: cuidado é fundamental, 2020; 12(2):1283-1289

2. BRAGANÇA GMG, et al. Evaluation of anxiety and depression prevalence in patients with primary severe hyperhidrosis. An Bras Dermatol, 2014; 84(2): 230-5.2014.

3. CAMPOS JJG, et al. Impact of primary hyperhidrosis on the life of physical education academics of a university in the Brazilian Northeast.Motriz: Rev educ fis, 2019; 25(2): e101914.

4. CAMPOS JRM, et al. Questionário de qualidade de vida em pacientes com hiperidrose primária. Jornal Brasileiro de Pneumologia, 2003; 29(4): 78-81.

5. DIAS LIN, et al. Relationship between anxiety, depression and quality of life with the intensity of reflex sweating after thoracoscopic sympathectomy for treatment of primary hyperhidrosis. Revista do Colégio Brasileiro de Cirurgiões, 2016; 43(5): 354-359.

6. DOOLITTLE J, et al. Hyperhidrosis: an update on prevalence and severity in the United States. Archives of Dermatological Research, 2016; 308(10): 743-749.

7. ESTEVAN FA, et al. Epidemiologic analysis of prevalence of the hyperhidrosis. Anais Brasileiros Dermatologia, 2017; 92(5): 630-634.

8. FELINI R, et al. Prevalência de hiperidrose em uma amostra populacional de Blumenau - SC, Brasil. Anais Brasileiros Dermatologia, 2009; 84(4):361-366.

9. FERREIRA FG, et al. Perda Eletrolítica de cálcio, magnésio e ferro no suor durante corrida em esteira. Revista Brasileira de Medicina e Esporte, 2017; 23(1): 31-36.

10. FIORELLI RKA, et al. Avaliação do Impacto na Qualidade de Vida de Pacientes Portadores de Hiperidrose Primária Submetidos à Simpatectomia Videotoracoscópica. Rev Meta Aval, 2011; 3(7):1-24.

11. HASIMOTO EN, et al. Hyperhidrosis: prevalence and impact on quality of life. Jornal Brasileiro de Pneumologia, 2018; 44(4): 1-7.

12. HONR, G.Criação e validação de um questionário de qualidade de vida para pacientes com Hiperidrose Primária, 2015. 83f. Dissertação( Residência Médica em Dermatologia).

13. LIMA SO, et al. Infraareolar Access for thoracoscopic sympathectomy to treat primary hyperhidrosis, Surgery Today, 2012; 43(2): 221-224.

14. LIMA SO, et al. Perfil Epidemiológico e Qualidade de Vida dos Estudantes de Medicina Portadores de Hiperidrose Primária. Revista Brasileira de Educação Médica, 2019; 43(1): 386 - 394.

15. LIMA SO, et al. Prevalência de hiperidrose primaria e fatores associados em uma capital do nordeste brasileiro: estudo baseado em população. Revista Eletrônica Acervo Saúde, 2020; 12(9): e3815.

16. LIMA SO, et al. Research of primary hyperhidrosis in students of medicine of the State of Sergipe, Brazil. Anais Brasileiros Dermatologia, 2015; 90(5): 661-665.

17. LIMA SO, et al. Retroperitoneoscopic lumbar sympathectomy for plantar hyperhidrosis. Journal of Vascular Surgery, 2017; 66(6): 1806-1813.

18. LIMA SO, SANTANA VR. The prevalence of hyperhidrosis worldwide. In Loureiro, M.; CAMPOS, J.; WOLOSKER N.; KAUFFMAN P, editors. Hyperhidrosis, Chan: Springer; 2018. p.33-38.

19. MIOTTO A, et al. Estudo comparativo da perda transepidérmica de água em pacientes com e sem hiperidrose por mensurador de câmara fechada em ambiente climatizado. Einstein (São Paulo), 2018; 16(4): 1-5.

20. MIRKOVIC SE, et al. Hyperhidrosis Substantially Reduces Quality of Life in Children: A Retrospective Study Describing Symptoms, Consequences and Treatment with Botulinum Toxin. Acta Dermato Venereologica, 2018; 98(1): $103-107$.

21. MORAES DKT, ROCHA GK. A hiperidrose no contexto escolar. Educação em Debate, 2018; 40(76): 9-25.

22. MORARD MRS, et al. Primary hyperhidrosis prevalence and characteristics among medical students in Rio de Janeiro. PLoS One, 2019; 14(9).

23. MURARO CSP, et al. Botulinum Toxin Type A in the Treatment of Hyperhidrosis: Case Study. Weber Medicine \& Clinical Case Reports, 2019; 7(1): 1247-1253.

24. MUTHUSAMY A, et al. A Study on the Impact of Hyperhidrosis on the Quality of Life among College Students. Journal of Clinical and Diagnostic Research, 2016; 10 (6): CC08 - CC10.

25. REIS GDD, et al.Estudo de pacientes com hiperidrose, tratados com toxina botulínica: análise retrospectiva 10 anos. Revista Brasileira de Cirurgia Plástica, 2011; 26(4)1.

26. RO KM, et al. Palmar hyperhidrosis: Evidence of genetic transmission. Journal of Vascular Surgery, 2002; 35(2): 382386.

27. SAKIYMA PY, B. et al. Avaliação quantitativa da intensidade da transpiração palmar e plantar em pacientes portadores de hiperidrose palmoplantar primária. J. bras. Pneumol, 2012; 38(5): 573-578.

28. SILVA SLS, et al. Avaliação da qualidade de vida de pacientes portadores de hiperidrose primária submetidos à simpatectomia videotoracoscópica. Revista do Colégio Brasileiro de Cirurgiões, 2017; 44(4): 323-327.

29. TOSCANO L, et al. Simpaticectomía torácica bilateral por videocirugía. Revista Médica del Uruguay, 2019; 35(1): 2026.

30. TU Y-R, et al. Epidemiological survey of primary palmar hyperhidrosis in adolescent in Fuzhou of People's Republic of China. European Journal of Cardio-thoracic Surgery, 2007; 31(4): 737-739.

31. VARELLA AYM, et al. Translation and validation of Hyperhidrosis Disease Severity Scale. Revista da Associação Médica Brasileira, 2016; 62(9): 843-847.

32. VASCONCELOS CFM, et al. Simpatectomia bilateral R5-R8 no tratamento de Hiperidrose compensatória: analises de complicações e satisfações dos pacientes. Revista do Colégio Brasileiro de Cirurgiões, 2020; 47: e20202398.

33. WESTPHAL FL, et al. Prevalência de hiperidrose entre estudantes de medicina. Revista do Colégio Brasileiro de Cirurgiões, 2011; 38(6): 392-397.

34. WOLOSKER N, et al. Current treatment options for craniofacial hyperhidrosis. Jornal Vascular Brasileiro, 2020; 19: e20190152.

35. WOLOSKER N, FAKUDA JM. O tratamento atual da hiperhidrose. Jornal Vascular Brasileiro, 2015; 14(4): $279-281$. 